\title{
Effets du méthomyl sur les tissus de maïs (Zea Mays L.) à cytoplasme normal ou à cytoplasme Texas en culture in vitro
}

\author{
B. Houndonougbo ${ }^{* 1}$ et $A$. Bervillé 2 \\ 1 Université nationale du Bénin, faculté des sciences et techniques, laboratoire de biologie végétale, Abomey-Calavi, BP 526 \\ Cotonou, Bénin; \\ 2 INRA, station d'amélioration des plantes, BV 1540, 21034 Dijon Cedex, France
}

(reçu le 5 janvier 1988, accepté le 30 mai 1989)

\begin{abstract}
Résumé - Les tissus mis en culture in vitro sont des scutellums immatures, et des rondelles de coléoptile de maïs à cytoplasme normal ou à cytoplasme Texas. Les tissus de maïs à cytoplasme normal sont résistants alors que ceux à cytoplasme Texas sont sensibles au méthomyl. Le méthomyl inhibe l'initiation et la croissance des cals des tissus à cytoplasme Texas alors que celles des tissus à cytoplasme normal ne sont pas affectées (Tableau II; Fig. 1). L'inhibition de la croissance des tissus à cytoplasme Texas sous l'action de 3,6 mM de méthomyl apparaît létale et irréversible tandis que les tissus à cytoplasme normal continuent à croître comme les tissus non traités au méthomyl. La sensibilité au méthomyl se révèle un marqueur spécifique du cytoplasme Texas.

La régénération de plantes a été réalisée chez les génotypes à cytoplasme normal : A188N, W64AN, F7N ou à cytoplasme Texas : A188T, W64AT et F7T, à partir des cals issus de scutellums immatures ou de rondelles de coléoptile de 1 à $2 \mathrm{~mm}$ d'épaisseur (Tableau III; Fig. 2). Les plantes à cytoplasme normal, régénérées sur le milieu de base (Tableau 1), contenant $36 \mu \mathrm{M}$ de méthomyl et $4,5 \mu \mathrm{M}$ de $2,4-\mathrm{D}$ ou $3,6 \mu \mathrm{M}$ de méthomyl et $0,45 \mu \mathrm{M}$ de $2,4 \mathrm{D}$, sont mâlefertiles et résistantes au méthomyl et les plantes à cytoplasme Texas néoformées, dans les mêmes conditions, sont demeurées mâle-stériles cytoplasmiques et sensibles au méthomyl.
\end{abstract}

\section{méthomyl - Zea mays L. - cytoplasme normal - cytoplasme Texas}

Summary - Effects of methomyl on the tissues of maize (Zea mays L.) with normal cytoplasm or Texas cytoplasm in in vitro culture. The tissues culture in vitro were immature scutellums, and coleoptile round slices of maize with normal or Texas cytoplasm. The maize tissues with normal cytoplasm were resistant while those with Texas cytoplasm were sensitive to methomyl. Methomyl inhibited the initiation and growth of the calluses from tissues with Texas cytoplasm, whereas those with normal cytoplasm were not affected (Table II; Fig. 1). The inhibition of the growth of Texas cytoplasm tissues under the action of $3.6 \mathrm{mM}$ methomyl appeared lethal and irreversible while normal cytoplasm tissues continued to grow in the same manner as those not treated with methomyl. The sensitivity to methomyl appears as a specific marker for the Texas cytoplasm.

The regeneration of plants has been carried out in the genotypes with normal-A188N, W64AN, F7N-or Texas cytoplasm : A188T, W64T, F7T from calli formed on immature scutellums or coleoptile round slices 1 to $2 \mathrm{~mm}$ thick (Table III; Fig. 2). The regenerated plants with normal cytoplasm on the basic medium (Table I) containing $36 \mu M$ of methomyl and $4.5 \mu \mathrm{M}$ of 2.4-D or 3.6 $\mu \mathrm{M}$ of methomyl and $0.45 \mu \mathrm{M}$ of 2.4-D are male-fertile and resistant to methomyl, while the neoformed plants with Texas cytoplasm under the same conditions, remained cytoplasmic male-sterile and sensitive to methomyl.

methomyl - Zea mays L. - normal cytoplasm - Texas cytoplasm

\section{INTRODUCTION}

Les toxines spécifiques ont fait l'objet de récentes investigations grâce à la technique de culture in vitro. C'est ainsi, par exemple, que Gengenbach \& Green (1975) ont obtenu des cals résistants à Helminthosporium maydis race $\mathrm{T}$, en mettant en culture des scutellums de maïs MSC-T sur un milieu synthétique contenant la toxine partiellement purifiée; que Person \& Horner (1976) ont observé le ralentissement de la croissance des cals, issus de mésocotyle de plantes mâle-stériles cytoplasmiques Texas (MSC-T) lorsqu'ils ont ajouté au milieu de culture la toxine d'H. maydis race T. De même Gengenbach et al. (1977) ont cultivé des scutellums de plantes MSC-T en présence de toxine d' $H$. maydis race $T$ et ont régénéré, après 12 cycles de

\footnotetext{
" Nouvelle adresse : 11/3/12, rue Ronsard, 59650 Villeneuve d'Asq, France.
} 
repiquages, 65 plantes résistantes dont 13 sont mâle-stériles. Les 52 autres plantes sont entièrement fertiles. Jaubertie et al. (1978) ont effectué des recherches de variants résistants à la T-toxine par la technique de culture de cellules et de tissus végétaux, sans succès pour les génotypes utilisés.

Les études réalisées par Humaydan \& Scott (1977); Berville (1979) et Houndonougbo (1984) ont montré la toxicité du méthomyl vis-à-vis du cytoplasme Texas : sur feuilles, sur tissus en culture, sur protoplastes et sur mitochondries isolées. En absence de méthomyl la prolifération des cellules de plantes Texas est comparable à celle des plantes à cytoplasme normal. En revanche, en présence de méthomyl, la prolifération des cellules Texas est inhibée alors que celle de plantes à cytoplasme normal n'est pas affectée (Houndonougbo, 1984).

II nous a alors paru intéressant d'étudier les effets de différentes concentrations de méthomyl sur la prolifération cellulaire (callogenèse) et le phénomène de régénération de plantes manifestés par les tissus de maïs traités par le carbamate méthomyl.

\section{MATÉRIEL ET MÉTHODES}

\section{Matériel végétal utilisé}

Toutes les semences certifiées des génotypes de maïs utilisés proviennent de la station d'amélioration des plantes de l'INRA de Clermont-Ferrand, du domaine Brunehaut, Estrées-Mons-de-Péronne, de la station expérimentale du maïs de Saint-Martin-de-Hinx et de la Société française des semences de maïs. Nous avons utilisé pour nos expériences les lignées A188N, W64AN, F7N mâle-fertiles sur cytoplasme normal ainsi que ces mêmes lignées converties sur cytoplasme mâle-stérile Texas (A188T, W64T, F7T).

\section{Méthodes}

Le méthomyl a été recristallisé du lannate selon la méthode décrite par Aranda et al. (1981). Nous avons incorporé au milieu de base (Tableau I) différentes concentrations : $0 \mathrm{M}, 3,6 \mu \mathrm{M}, 36 \mu \mathrm{M}, 0,36 \mathrm{mM}, 3,6 \mathrm{mM}$ de méthomyl et $0 \mathrm{M}, 0,045 \mu \mathrm{M}, 0,45 \mu \mathrm{M}, 4,5 \mu \mathrm{M}, 9 \mu \mathrm{M}$ d'acide 2,4-dichlorophénoxyacétique (2,4-D). Ce milieu est autoclavé à $115^{\circ} \mathrm{C}$ durant 20 min après ajustement du $\mathrm{pH}$ à 5,8 à l'aide de soude $0,1 \mathrm{~N}$. II est ensuite coulé en boîtes de Petri stériles en plastique de 55 ou $90 \mathrm{~mm}$ de diamètre.

Les plantes mères sur lesquelles les scutellums immatures sont prélevés sont cultivées en serre où la température est d'environ $25^{\circ} \mathrm{C}$ le jour et $18^{\circ} \mathrm{C}$ la nuit. Les épis sont prélevés à partir du $14{ }^{\circ}$ jusqu'au $26^{\circ}$ jour après la pollinisation manuelle. Après ablation des spathes, les épis sont stérilisés dans une solution d'hypochlorite de calcium à $7 \%$ durant 20 min puis rincés 3
Tableau I. Milieu de base.

Macro-élements : Murashige et Skoog (1962)

Micro-éléments : Murashige et Skoog (1962)

Acide nicotinique

Pyrodoxine $\mathrm{HCl}$

Thiamine $\mathrm{HCl}$

Méso-inositol

Glycine

Saccharose

Agar

$5 \mathrm{mg} \mathrm{|}^{-1}$

$1 \mathrm{mg} \mathrm{l}^{-1}$

$1 \mathrm{mg} \mathrm{l}^{-1}$

$100 \mathrm{mg} \mathrm{l}^{-1}$

$2 \mathrm{mg} \mathrm{l}^{-1}$

$20 \mathrm{mg} \mathrm{l}^{-1}$

$8 \mathrm{mg} \mathrm{l}^{-1}$

fois à l'eau distillée stérile. Les scutellums immatures sont prélevés de 14 à 26 jours après la fécondation dans la partie médiane de l'épi.

Pour obtenir des coléoptiles, des grains secs et mûrs sont trempés dans de l'alcool à $70^{\circ}$ pendant 10 min, ensuite dans l'hypochlorite de calcium à $7 \%$ pendant $20 \mathrm{~min}$. Ils sont rincés 3 fois à l'eau distillée stérile. Ils sont réhydratés dans de l'eau distillée stérile pendant $72 \mathrm{~h}$ en agitation rotative ( 70 tours $/ \mathrm{min}$ ) à $25 \pm 1^{\circ} \mathrm{C}$ à la lumière du jour. Ils sont ensuite rincés 3 fois à l'eau distillée stérile et cultivés en condition stérile sur un milieu solide ne contenant que de l'eau et $0,8 \%$ d'agar. Après 4 jours de culture sur ce milieu dans une étuve à $25 \pm 1^{\circ} \mathrm{C}$ à la lumière avec une photopériode de $8 \mathrm{~h}$, une intensité lumineuse d'environ $2500 \mathrm{erg} \mathrm{cm}^{2} \mathrm{~s}^{-1}$ (tubes Sylvania Grolux), les coléoptiles sont prélevés et débités en rondelles d'environ 1 à $2 \mathrm{~mm}$ d'épaisseur.

Les scutellums immatures, soit 216 scutellums par génotype, et les rondelles de coléoptiles sont ensemencés en boîte de Petri contenant du milieu de culture. Les boîtes de Petri sont serties de bandes de Parafilm et sont alors portées dans une salle de culture à $25 \pm 1{ }^{\circ} \mathrm{C}$, à la lumière avec une photopériode de $16 \mathrm{~h}$ et une intensité lumineuse d'environ $2500 \mathrm{erg} \mathrm{cm}^{2} \mathrm{~s}^{-1}$ au niveau des explants.

Après 15 jours de culture, des scutellums Texas traités par 3,6 mM de méthomyl sont rincés 3 fois à l'eau distillée stérile afin d'éliminer le méthomyl. Ils sont ensuite transférés sur le milieu de base, neuf, contenant seulement $9 \mu \mathrm{M}$ de 2,4-D. Après un mois de culture, le rythme de croissance permet un cycle de repiquage des cals verts embryogènes en 15 jours. Nous avons déterminé le poids de matière sèche en mg des explants, une fois le rythme de croissance atteint, soit après le premier mois de culture.

En fin de culture, nous avons procédé au dénombrement des plantes régénérées.

Nous avons éprouvé la résistance en cours de végétation à l'aide de la scarification des feuilles de jeunes plantes régénérées de maïs cultivées en serre et du dépôt sur la blessure de $10 \mu \mathrm{l}$ de $0,123 \mathrm{M}$ de méthomyl et sur la blessure servant de témoin $10 \mu \mathrm{l}$ d'eau distillée.

\section{RÉSULTATS}

\section{Callogenèse}

L'addition de 3,6 mM de méthomyl au milieu de base (Tableau I) inhibe complètement la crois- 
sance des scutellums immatures des génotypes de maïs à cytoplasme Texas : A188T et W64AT tandis que, dans les mêmes conditions, la croissance des scutellums des génotypes à cytoplasme normal : $A 188 \mathrm{~N}$ et $W 64 \mathrm{~N}$ est analogue à celle des tissus témoins, non traités par le méthomyl (Tableau II).

Le poids des cals obtenus sur les scutellums immatures de plantes Texas après un mois de culture sur le milieu de base contenant 3,6 mM de méthomyl avec ou sans 2,4-D est nul. Les doses plus faibles de méthomyl - 0,36 mM, 36 $\mu \mathrm{M}, 3,6 \mu \mathrm{M}$ - retardent l'apparition des cals ainsi que leur croissance ultérieure.

Le méthomyl bloque, dès le début de son application, la multiplication cellulaire (Tableau II; Fig. 1). Après 15 jours de culture sur le milieu de base contenant $3,6 \mathrm{mM}$ de méthomyl et $9 \mu \mathrm{M}$ de 2,4-D, 72 scutellums immatures Texas sont rin-

Poids moyen des cals tormes en $\mathrm{mg}$ de matiere seche par scutellum immature.

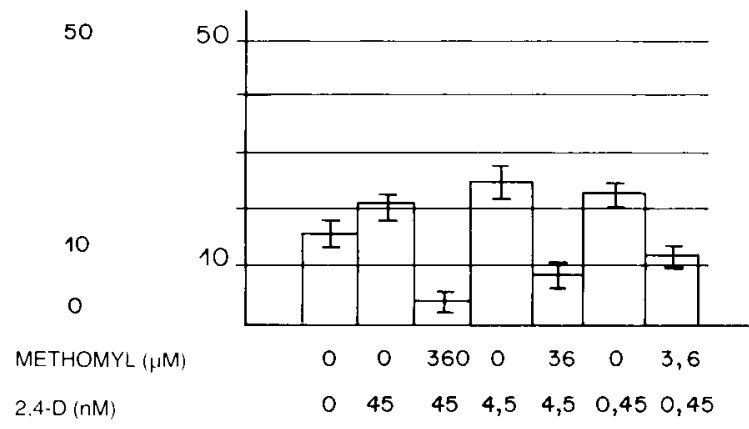

Fig. 1. Action du méthomyl et du 2,4-D sur la callogenèse des scutellums immatures de maïs à cytoplasme Texas (A188T). I = intervalle de confiance. cés 3 fois à l'eau distillée stérile et sont transférés sur un milieu de base, neuf, renfermant $9 \mu \mathrm{M}$ de 2,4-D sans méthomyl. Ils ne croissent pas, après un mois de culture sur ce dernier milieu (nion montré). En revanche, dans les mêmes conditions de culture, les scutellums immatures à cytoplasme normal : A188N et W64AN continuent à croître comme les tissus témoins traités par le méthomyl.

Le méthomyl n'inhibe pas la croissance des tissus à cytoplasme normal. Ces tissus sont résistants au méthomyl. Les effets du méthomyl sur les tissus de maïs à cytoplasme Texas sont spécifiques.

\section{Régénération de plantes}

\section{Régénération de plantes à partir des ron- delles de coléoptile}

Des rondelles de 1 à $2 \mathrm{~mm}$ d'épaisseur de coléoptile de maïs en culture in vitro sur le milieu de base (Tableau I) contenant du méthomyl et du 2,4-D initient des cals, mais très souvent, la plupart forment rapidement des racines et développent des plantes entières (Tableau III; Fig. 2). Cette forme de multiplication végétative in vitro se réalise aisément chez les génotypes de maïs à cytoplasme normal : A188N, W64AN et F7N. En revanche, chez les génotypes à cytoplasme Texas : A188T, W64AT et F7T, quelques plantes ont pu être régénérées seulement pour les très faibles doses de méthomyl $36 \mu \mathrm{M}$ et $3,6 \mu \mathrm{M}$ (Tableau III). Les plantes néoformées ont été repiquées en terre à la serre. Celles qui ont pu se développer ont fleuri et ont produit des grains. Les plantes néoformées à cytoplasme normal

Tableau II. Indication du poids moyen de cal formé en $\mathrm{mg}$ de matière sèche par scutellum immature pour un mois de culture (intervalle de confiance $\alpha=5 \%$ ).

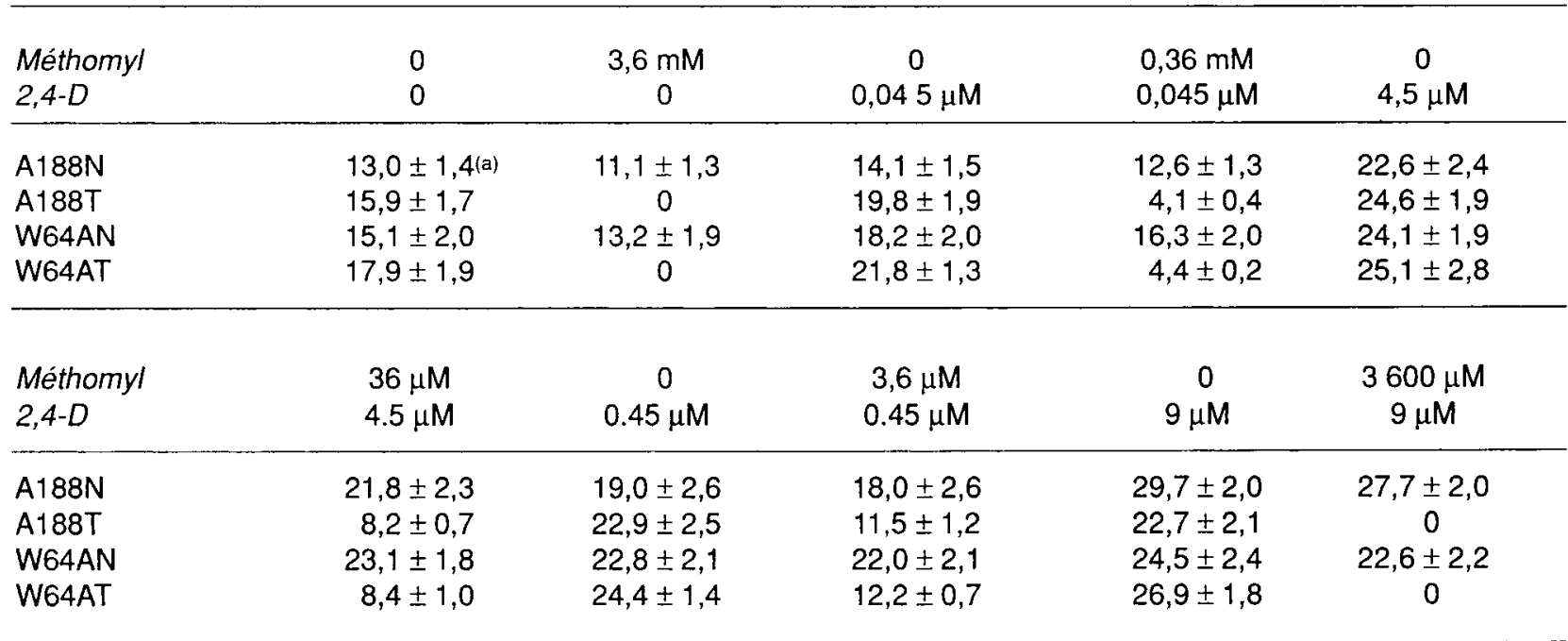

(a) : 216 scutellums par répétition. 
Tableau III. Indication du nombre de plantes régénérées par milieu et par génotype à partir de rondelles de coléoptile de maïs à cytoplasme normal $(\mathrm{N})$ ou à cytoplasme Texas $(\mathrm{T})$ et total des plantes régénérées par génotype.

\begin{tabular}{|c|c|c|c|c|c|c|}
\hline $\begin{array}{l}\text { Méthomyl } \\
2,4-D\end{array}$ & $\begin{array}{l}0 \\
0\end{array}$ & $\begin{array}{c}0 \\
0,045 \mu \mathrm{M}\end{array}$ & $\begin{array}{c}0 \\
0,45 \mu \mathrm{M}\end{array}$ & $\begin{array}{c}0 \\
4,5 \mu \mathrm{M}\end{array}$ & $\begin{array}{c}0 \\
9 \mu \mathrm{M}\end{array}$ & Total (1) \\
\hline A188N & 19 & 18 & 17 & 13 & 5 & 72 \\
\hline A188T & 15 & 11 & 8 & 5 & 3 & 42 \\
\hline W64AN & 7 & 6 & 3 & 2 & 1 & 19 \\
\hline W64AT & 18 & 15 & 7 & 6 & 3 & 49 \\
\hline F7N & 3 & 2 & 1 & 1 & 1 & 8 \\
\hline F7T & 10 & 9 & 5 & 3 & 1 & 28 \\
\hline Méthomyl & 0 & $0,36 \mathrm{mM}$ & $36 \mu \mathrm{M}$ & $3,6 \mu \mathrm{M}$ & $3,6 \mu \mathrm{M}$ & Total (1) \\
\hline $2,4-D$ & 0 & $0,045 \mu \mathrm{M}$ & $4,5 \mu \mathrm{M}$ & $0,45 \mu \mathrm{M}$ & $9 \mu \mathrm{M}$ & \\
\hline $\mathrm{A} 188 \mathrm{~N}$ & 19 & 8 & 5 & 6 & 1 & 39 \\
\hline A188T & 15 & 0 & 3 & 5 & 0 & 23 \\
\hline W64AN & 7 & 5 & 1 & 3 & 1 & 17 \\
\hline W64AT & 18 & 0 & 1 & 3 & 0 & 22 \\
\hline F7N & 3 & 1 & 1 & 1 & 1 & 7 \\
\hline F7T & 10 & 0 & 2 & 3 & 0 & 15 \\
\hline
\end{tabular}

(1): Total des plantes régénérées par génotype.

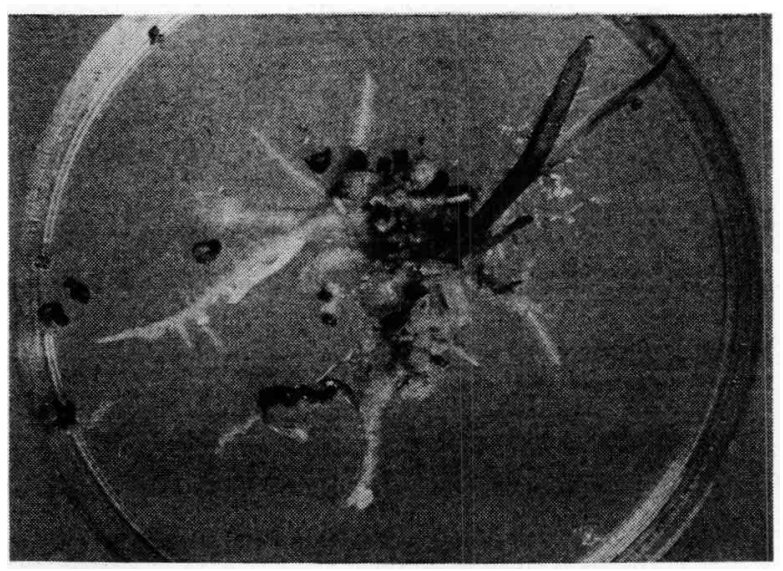

Fig. 2. Régénération de plante à partir d'une rondelle de 1 à 2 $\mathrm{mm}$ d'épaisseur de coléoptile de maïs (Zea mays L.) à cytoplasme Texas (A188T) en culture in vitro sur le milieu de Murashige et Skoog (1962) contenant 3,6 $\mu \mathrm{M}$ de méthomyl et $0,45 \mu \mathrm{M}$ de 2,4-D.

sont mâle-fertiles et résistantes au méthomyl et les plantes régénérées à cytoplasme Texas sont mâle-stériles cytoplasmiques et sensibles au méthomyl.

\section{DISCUSSION}

L'initiation des cals in vitro sur divers milieux sans toxine spécifique à partir de scutellums immatures, de fragments de mésocotyle et de coléoptile de maïs ainsi que la régénération de plantes entières ont été réalisées par Green \& Phillips (1975), Harms et al. (1976), Vuillaume \& Deshayes (1977), Raffail (1978), Beckert \& Pol- lacsek (1979), Torne et al. (1980). L'effet stimulant du 2,4-D sur la croissance est important.

Les résultats de notre étude sur les effets du méthomyl sur les tissus de maïs à cytoplasme normal ou à cytoplasme Texas nous suggèrent les idées suivantes.

En présence de méthomyl les tissus à cytoplasme Texas sont inhibés in vitro quel que soit le critère de croissance choisi, alors que ceux à cytoplasme normal continuent à croître. La spécificité in vitro du méthomyl est donc absolue, (Houndonougbo, 1984).

Nous observons que les doses de méthomyl qui ont un effet d'inhibition pour les tissus Texas sont de l'ordre de $36 \mu \mathrm{M}$. Le mode d'action supposé du méthomyl, reconnu dans les mitochondries Texas, serait de détruire le potentiel membranaire des mitochondries (Berville et al., 1085) et de provoquer un effet découplant (Klein \& Koeppe, 1985). Les doses actives de méthomyl sur les mitochondries Texas sont de l'ordre de $1 \mathrm{mM}$ à $3 \mathrm{mM}$ pour une inhibition totale.

Les tissus de maïs Texas en culture sont donc inhibés en quelques jours par des concentrations 10 à 15 fois plus faibles que celles qui ont un effet de diminution sur le couplage des mitochondries. On peut se demander alors si les tissus Texas accumulent le méthomyl. Les expériences réalisées par Berville et al. (1985) infirment cette hypothèse puisque aucune accumulation de méthomyl radioactif n'a été décelée dans les tissus sensibles ( $T$ ) par rapport aux tissus résistants $(\mathrm{N})$. Une autre hypothèse serait que le 
méthomyl ait un site d'action sur la membrane cellulaire d'affinité plus grande pour le méthomyl que la mitochondrie. Cette hypothèse, pour être éprouvée, demande une expérimentation que nous réalisons.

Les quelques plantes Texas régénérées sont sensibles au méthomyl; nous n'avions réalisé que 2 cycles de repiquage et obtenu que peu de plantes. Nous pensons maintenant que les conditions sont déterminées pour réaliser sur un effectif plus important la sélection de plantes résistantes au méthomyl comme cela a pu être fait avec la T-toxine d' $H$. maydis (Gengenbach et al., 1977 et Brettel et al., 1980).

\section{CONCLUSION}

Le méthomyl inhibe in vitro l'initiation et la croissance des cals des tissus à cytoplasme Texas, alors que celles des tissus à cytoplasme normal ne sont pas affectées. La toxicité du méthomyl s'accompagne rapidement d'effets létaux pour les tissus à cytoplasme Texas. L'inhibition de la croissance des tissus à cytoplasme Texas sous l'action de 0,36 mM de méthomyl apparaît totale et irréversible alors que les tissus à cytoplasme normal continuent à croître comme les tissus non traités au méthomyl (Houndonougbo, 1984). La spécificité de l'inhibition par le méthomyl de la croissance des tissus à cytoplasme Texas permet d'envisager de cribler des cellules ou des cals résistants. Pour cela il fallait déterminer la gamme de sensibilité in vitro. Nous avons constaté que les doses actives sont beaucoup plus faibles pour les cellules in vitro que pour les mitochondries isolées.

Les doses de méthomyl utilisées pour le criblage devront être très faibles de $100 \mu \mathrm{M}$ à $1 \mathrm{mM}$.

Pour expliquer la sensibilité des cellules in vitro, nous recherchons des modifications physiologiques induites par le méthomyl, la fuite d'un cofacteur le NAD a été détectée (Houndonougbo \& Bervillé, 1988).

\section{RÉFÉRENCES}

Aranda G., Bervillé A., Cassini R., Fetizon M. \& Poiret P. (1981) Etude de l'hydrolyse et des propriétés toxiques vis-à-vis des mitochondries de maïs Texas d'un insecticide, le méthomyl ou $S$-méthyl- $N$ [méthylcarbamoyl)oxyl] thioacétimidate. Experientia 37, 112113

Beckert M. \& Pollacsek M. (1979) Expression de la variabilité du maïs (Zea mays L.) en différentes conditions de culture de tissus. Ann. Amélior. Plantes 29, 563-581

Bervillé A. (1979) Etude préliminaire de la stérilité mâle cytoplasmique. Thèse de Doctorat d'Etat ès Sciences
Naturelles présentée à l'Université de Paris-Sud (XI), Centre d'Orsay, 1-173

Bervillé A., Schneider C., Bonavent J.F., Bodergat R. \& Aranda G. (1985) No accumulation of methomyl in texas maize mitochondria. International Symposium on "Achievements and Perspectives in Mitochondrial Research" 148, 114 (Abstract)

Brettell R.I.S., Thomas E. \& Ingram D.S. (1980) Reversion of Texas male-sterile cytoplasm maize in culture to give fertile, T-toxin resistant plants. Theor. Appl. Genet. 58, 55-58

Gengenbach B.G. \& Green C.E. (1975) Selection of T-cytoplasm maize callus cultures resistant to Helminthosporium maydis race $T$ pathotoxin. Crop. Sci. 15, 645-649

Gengenbach B.G., Green C.E. \& Donovan C.M. (1977) Inheritance of selected pathotoxin resistance in maize plants regenerated from cell cultures. Proc. Natl. Acad. Sci. USA 74, 5113-5117

Green C.E. \& Phillips R.L. (1975) Plant regenerated from tissue culture of maize. Crop Sci. 15, 417-421

Harms C.E., Lorz H. \& Potrykus J. (1976) Regeneration of plantlets from callus cultures of Zea mays $L$. $Z$. Pflanzenzuchtg. 77, 347-351

Houndonougbo B. (1984) Contribution au mode d'action d'un carbamate : le méthomyl, par la réaction des protoplastes et culture de tissus de maïs (Zea mays L.) à cytoplasme normal ou à cytoplasme Texas. Thèse de Doctorat d'Etat ès Sciences Naturelles présentée à l'Université de Paris-Sud (XI), Centre d'Orsay, 1-201

Houndonougbo B. \& Bervillé A. (1988) Effets du méthomyl sur la libération du nicotinamide adénine nucléotide des protoplastes de maïs (Zea mays L.) à cytoplasme normal ou à cytoplasme Texas en culture in vitro. C.R. Acad. Sci. Paris, Sér. III 307, 459-464

Humaydan D.S. \& Scott E.W. (1977) Methomyl insecticide selective phytotoxicity on sweet corn hybrids and inbreds having the Texas male-sterile cytoplasm. Hort. Sci. 12, 312-320

Jaubertie J.P., Lafouasse M., Bervillé A., Branchard M., Cassini R., Cornu A., Deshayes A. \& Vuillaume E. (1978) Recherches de variants résistants à des toxines par la technique de culture de cellules et de tissus végétaux. Physiol. Vég. 16, 401-409

Klein R.R. \& Koeppe D.E. (1985) Mode of methomyl and Bipolaris maydis (race $T$ ) toxin in uncoupling Texas male-sterile cytoplasm corn mitochondria. Plant Physiol. 77, 912-916

Murashige T. \& Skoog F. (1962) A revised medium for rapid growth and bioassays with tobacco tissue cultures. Physiol. Plant 15, 473-497

Person C.B. \& Horner H.T. (1976) Callus formation and differentiation in tissue cultures of normal and Texas cytoplasmic male sterile corn. Proc. Towa Acad. Sci. 82, 163-165

Raffail W.S. (1978) The initiation of callus tissue from immature embryos of maize. Maize Genet. Coop. News Lett. 52, 3-5

Torne J.M., Santos M.A., Pons A. \& Blanco M. (1980) Regeneration of plants from mesocotyl tissue cultures of immature embryos of Zea mays. Plant Sci. Lett. 17, 339-344

Vuillaume E. \& Deshayes A. (1977) Initiation des cals in vitro à partir de fragments de mésocotyle et de coléoptile chez le maïs. Ann. Amélior. Plantes 27, 657673 